\title{
A NOTE ON THE STRUCTURE OF MATRIX *-SUBALGEBRAS WITH SCALAR DIAGONALS
}

\author{
G. MACDONALD ${ }^{1}$, L. W. MARCOUX ${ }^{1}$, M. MASTNAK ${ }^{1}$, \\ M. OMLADIČ ${ }^{2}$ AND H. RADJAVI
}

Abstract. We characterize those unital, self-adjoint algebras of complex $n \times n$ matrices that are simultaneously unitarily similar to algebras in which every member has a scalar diagonal.

Mathematics subject classification (2010): 15A04, 15A21.

Keywords and phrases: Unitary similarity, scalar diagonals, self-adjoint subalgebras of matrices.

\section{REFERENCES}

[1] P. FAn, On the diagonal of an operator, Trans. Amer. Math. Soc. 283, no. 1: 239-251, 1984.

[2] P. A. Fillmore, On similarity and the diagonal of a matrix, Amer. Math. Monthly 76: 167-169, 1969.

[3] P. R. Halmos, Finite Dimensional Vector Spaces, Van Nostrand, Princeton, N.J., 1958.

[4] A. HoRn, Doubly stochastic matrices and the diagonal of a rotation, Amer. J. Math. 76: 620-630, 1954.

[5] L. Mirsky, Matrices with prescribed characteristic roots and diagonal elements, J. London Math. Soc. 33: 14-21, 1958. 